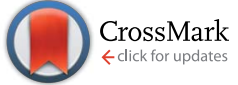

Cite this: RSC Adv., 2017, 7, 208
Received 14th October 2016 Accepted 3rd November 2016

DOI: $10.1039 / c 6 r a 25245 a$

www.rsc.org/advances

\title{
Optimized preparation of micro-block CPAM by response surface methodology and evaluation of dewatering performance $\uparrow$
}

\author{
Xiang Li, ${ }^{\text {ab }}$ Huaili Zheng, ${ }^{\star a b}$ Baoyu Gao, ${ }^{c}$ Yongjun Sun, ${ }^{d}$ Xiaomin Tang ${ }^{e}$ \\ and Bincheng $\mathrm{Xu}^{\mathrm{ab}}$
}

\begin{abstract}
Micro-block cationic polyacrylamide (P(AM-MAPTAC)) was synthesized through UV-initiated template copolymerization and characterized by FTIR, ${ }^{1} \mathrm{H}$ NMR, SEM and TG/DSC analyses. Furthermore, the main influence factors of the preparation process were optimized through Box-Behnken experiment design and Respond Surface Method (RSM). The maximum intrinsic viscosity of micro-block CPAM was 13.223 $\mathrm{dL} \mathrm{g}^{-1}$ under the optimum synthesis conditions. The dewatering performance of micro-block CPAM was evaluated taking activated sludge collected from a dyeing mill as the processing object. Results showed that the micro-block CPAM exhibited excellent dewatering performance. At a $30 \mathrm{mg} \mathrm{L}^{-1}$ dosage of micro-block CPAM, the residual turbidity, filter cake moisture content, specific resistance to filtration and chemical oxygen demand removal rate reached $4.47 \mathrm{NTU}, 72.2 \%, 5.47\left(10^{12} \mathrm{~m} \mathrm{~kg}{ }^{-1}\right)$ and $79.2 \%$, respectively. Dewatering tests not only demonstrated the superiority of micro-block P(AM-MAPTAC) synthesized by UV-initiated template copolymerization over that synthesized by traditional methods but also demonstrated that increasing the molecular weight can further enhance the dewatering performance of flocculants.
\end{abstract}

\section{Introduction}

With the increasing development of the textile industry, dyeing wastewater discharge increases year by year, and a large amount of excess sludge is ultimately produced. Excess sludge disposal has become a major factor restricting the development of the textile industry. Dyeing excess sludge with a high moisture content, complex composition, and high content of harmful substances is categorized as hazardous solid waste. ${ }^{\mathbf{1} 2}$ It is of very important significance to realize a harmless, reduction and stabilization treatment of dyeing excess sludge along with the increase of environmental awareness and strict environmental regulations. In the process of dyeing excess

${ }^{a}$ Key Laboratory of the Three Gorges Reservoir Region's Eco-Environment, State Ministry of Education, Chongqing University, Chongqing 400045, China. E-mail: lx33cqu@163.com; zhl6512@126.com; Fax:+8623 65120827; Tel: +862365120827 ${ }^{b}$ National Centre for International Research of Low-carbon and Green Buildings, Chongqing University, Chongqing 400045, China

${ }^{c}$ Shandong Key Laboratory of Water Pollution Control and Resource Reuse, School of Environmental Science and Engineering, Shandong University, Ji'nan 250100, China ${ }^{d}$ Jiangsu Key Laboratory of Industrial Water-Conservation \& Emission Reduction, College of Urban Construction, Nanjing Tech University, Nanjing, 211800, China

${ }^{e}$ Chongqing Key Laboratory of Catalysis and Environmental New Materials, College of Environment and Resources, Chongqing Technology and Business University, Chongqing 400067, P. R. China

$\dagger$ Electronic supplementary information (ESI) available. See DOI: 10.1039/c6ra25245a sludge treatment, the moisture content of the concentrated sludge is more than $97 \% .^{3}$ Therefore, dewatering is the most important means of sludge reduction in the whole sludge treatment system. Excess sludge is a colloidal system in which fine sludge particles are suspended in water owing to electrostatic repulsion and is difficult to condense to form a large particle size to facilitate subsequent dewatering process., ${ }^{\mathbf{4} 5}$ Therefore, before the mechanical dewatering, it is necessary to adjust the sludge properties to enhance its dewatering performance.

Cationic polyacrylamide (CPAM) is a kind of synthetic organic compound with excellent sludge dewatering performance. ${ }^{6,7}$ Positive charged CPAM can effectively neutralize the negative charge on the surface of sludge. Furthermore, long chain molecular structure is conducive to adsorption and bridging effect in the coagulation-flocculation process. Relative molecular weight and cationic degree are considered to be the two most important parameters affecting the flocculation performance of CPAM. Relative molecular weight (limiting viscosity) directly affects the efficiency of bridging effect., Therefore, to improve the relative molecular weight $\left(M_{\mathrm{W}}\right)$ of polymer has become an important research direction in CPAM synthesis. ${ }^{\mathbf{1 0 - 1 2}}$ The cationic degree (CD) is closely related to the electrical neutralization performance of CPAM. Besides, CD can also affect the morphology of CPAM in aqueous solution. The strength of the electrostatic repulsion between the positive charges in the CPAM molecular chain can affect the extension of 
the CPAM molecular chain in water. ${ }^{13-15}$ However, due to the higher price of the cationic monomer, it is uneconomical to enhance the dewatering performance of CPAM only by increasing the CD. In recent years, researchers have begun to pay attention to the effect of CPAM sequence structure distribution on flocculation performance and some studies have indicated that polymer with cationic micro-block structure is more conducive to the coagulation-flocculation process. ${ }^{\mathbf{1 6}-19}$ Our previous works have also proved the cationic micro-block structure in CPAM chain is conducive to the electrical neutralization and thus enhance the dewatering performance of CPAM, furthermore, verified the feasibility of synthesizing CPAM with micro-block structure by UV-initiated mode in combination with template polymerization technology. ${ }^{20,21}$ However, the CPAM with cationic micro-block structure synthesized in our previous works were all with lower molecular weight and thus with a weaker adsorption bridging effect. Therefore, in order to make CPAM play both strong electrical neutralization and adsorption bridging effect in coagulation-flocculation process, it is necessary to optimize the preparation conditions of CPAM to obtain CPAM with both cationic micro-block structure and high relative molecular.

Response surface analysis (RSM) is a statistical method for process design and parameter optimization. In this method, the continuous variable surface model is established to evaluate the factors that affect the reaction process and their interaction, so as to obtain the best experimental conditions. This method has the advantages of short experimental period and can directly reflect the optimal value of the dependent variable, thus received wide attention and extensive application. Hence, this method was used to optimize the synthesis conditions of microblock CPAM in this study.

In the present study, CPAM with micro-block was synthesized by UV-initiated template polymerization. The properties of micro-block CPAM were tested by using Fourier transform infrared spectroscopy (FTIR), ${ }^{1} \mathrm{H}$ nuclear magnetic resonance $\left({ }^{1} \mathrm{H}\right.$ NMR), scanning electron microscopy (SEM) and thermal analysis (TG/DSC). Box-Behnken experiment design and Respond Surface Method (RSM) were used to optimize the synthesis conditions of micro-block CPAM. Furthermore, flocculation performance of micro-block CPAM were explored in terms of residual turbidity (RT), filter cake moisture content (FCMC), specific resistance to filtration (SRF), chemical oxygen demand (COD) of supernatant and floc properties.

\section{Materials and methods}

\section{Materials}

Synthesis reagents. All the reagents used in the synthesis reaction were of analytical reagent grade except acrylamide (AM) and methacrylamido propyl trimethyl ammonium chloride (MAPTAC) (technical grade). The details of the materials are as follows: AM (Chongqing Lanjie Tap Water Company, Chongqing, China); MAPTAC (50 wt\% in water; Nanjing Jingruijiuan Biotechnology Co., Ltd. Nanjing, China); EDTA, urea, absolute ethyl alcohol and acetone (Chongqing Chuandong Chemical Industry Co., Ltd. Chongqing, China); VA-044 (Ruihong Biological Technology, Shanghai, China).

Dewatering reagents. All the flocculants used in the dewatering tests were synthesized in our laboratory except the commercial CPAM (technical grade, Chongqing Lanjie Tap Water Company, Chongqing, China). The details of flocculants are listed in Table 1 . The dyeing excess sludge was collected from sludge thickener of one dyeing mill in Chongqing China. The sludge featured $99.2 \%$ of moisture, $0.95 \mathrm{~kg} \mathrm{~L}^{-1}$ of density, 10.8 of $\mathrm{pH},-16.8$ of zeta potential, $8138 \mathrm{mg} \mathrm{\textrm {L } ^ { - 1 }}$ of COD and dark green, granular loose and pungent smell of apparent characteristic.

\section{Optimize synthesis conditions by RSM}

The synthesis process and characterization methods were described in S1 and S2 Texts. $\dagger$ An integrative method of BoxBehnken (BBD) and RSM was used to optimize the synthesis process of micro-block P(AM-MAPTAC), the experimental range for independent variables was preliminary trials based, with negligible error. In the optimization test, five independent variables, $\mathrm{pH}\left(X_{1}\right)$, concentration of initiator (wt\% of total monomer) $\left(X_{2}\right)$, mole ratio of PAAS and MAPTAC ( $n$ (PAAS/ MAPTAC)) $\left(X_{3}\right)$, mole ratio of MAPTAC and AM (n(MAPTAC/ $\mathrm{AM})$ ) $\left(X_{4}\right)$, irradiation time $(t / \mathrm{min})\left(X_{5}\right)$ were chosen for BBDRSM approach, while intrinsic viscosity $\left(\eta / \mathrm{dL}^{-1}\right)$ was considered as response variable. The details of BBD experimental design are listed in Table 2. $\mathrm{B}_{46}\left(3^{5}\right)$ was used to improve the experiment. As given in Table 2, eqn (1) was used to account for the control and prediction of optimization process.

$$
Y=\beta_{0}+\sum_{i=1}^{k} \beta_{i} X_{i}+\sum_{i=1}^{k} \beta_{i i} X_{i}^{2}+\sum_{i=1}^{k-1} \sum_{j=i+1}^{k} \beta_{i j} X_{i} X_{j}
$$

Table 1 Details of flocculants used in dewatering tests

\begin{tabular}{llrl}
\hline Flocculants & Full name of flocculants & Intrinsic viscosity dL g $^{-1}$ & Cationic degree (\%) \\
\hline TP1 & Template poly(AM-MAPTAC) 1\# & 9.050 & 25 \\
TP2 & Template poly(AM-MAPTAC) 2\# & 11.280 & 25 \\
TP3 & Template poly(AM-MAPTAC) 3\# & 13.223 & 25 \\
P $^{a}$ & P(AM-MAPTAC) & 13.348 & 25 \\
PPAM $^{b}$ & Commercial P(AM-DAC) & 13.386 & 25
\end{tabular}

${ }^{a}$ Flocculants P was synthesized by UV-initiated copolymerization without addition template PAAS. ${ }^{b}$ Flocculants CPAM was commercial product and synthesized taking DAC as the cation monomer. 
Table 2 Levels of the independent variables designed in the $\mathrm{B}_{46}\left(3^{5}\right)$

\begin{tabular}{lllll}
\hline & \multicolumn{5}{c}{ Levels } \\
\cline { 5 - 5 } $\begin{array}{l}\text { Independent } \\
\text { variables }\end{array}$ & Code & -1 & 0 & 1 \\
\hline $\mathrm{pH}$ & $X_{1}$ & 3 & 4.75 & 6.5 \\
Va-044 (wt\%) & $X_{2}$ & 0.02 & 0.07 & 0.12 \\
$n$ (PAAS/MAPTAC) & $X_{3}$ & 0.5 & 1 & 1.5 \\
$n$ (MAPTAC/AM) & $X_{4}$ & 0.15 & 0.33 & 0.5 \\
$t$ (min) & $X_{5}$ & 30 & 60 & 90 \\
& & & &
\end{tabular}

where $\beta_{0}, \beta_{i}, \beta_{i i}$ and $\beta_{i j}$ are regression coefficients, $X_{i}$ are coded independent variables. The designs contribute to minimize the unexpected effects and set up reasonable mathematical models for optimization process.

\section{Dewatering tests}

$1 \mathrm{~g} \mathrm{~L}^{-1}$ (concentration of pure flocculants) of flocculants were pre prepared by dissolving a certain amount of solid flocculants in $100 \mathrm{ml}$ distilled water. All the dewatering tests were conducted on a program-controlled jar-test apparatus (ZR4-6, Zhongrun Water Industry Technology Development Co., China) at room temperature. RT and COD of supernatant, FCMC and SRF of sludge were used to evaluate the dewatering performance of flocculants. In the sludge dewatering test, a set dosage of flocculants was added into $250 \mathrm{ml}$ beaker with $100 \mathrm{ml}$ initial dyeing excess sludge and the $\mathrm{pH}$ was adjusted with sodium hydroxide $\left(0.1 \mathrm{~mol} \mathrm{~L}^{-1}\right)$ and hydrochloric acid $(0.1 \mathrm{~mol}$ $\mathrm{L}^{-1}$ ) to the set value. Then quickly stirring at $120 \mathrm{rpm}$ for $20 \mathrm{~s}$ and followed by $40 \mathrm{rpm}$ for $60 \mathrm{~s}$, then stand for $10 \mathrm{~min}$. The supernatant $1 \mathrm{~cm}$ below the water surface was collected for the measurement of RT, COD and zeta potential, and then the conditioned sludge samples were filtered through a vacuum suction filter machine under a pressure of $0.05 \mathrm{MPa}$ for $30 \mathrm{~min}$ for the FCMC and SRF measure. The detail measure methods of RT, COD, zeta potential, FCMC and SRF are described in S3 Text. $\dagger$ The sludge settle performance was conducted in a $500 \mathrm{ml}$ graduated cylinder. $500 \mathrm{ml}$ original dyeing sludge with $30 \mathrm{mg}$ $\mathrm{L}^{-1}$ dosage of flocculants was firstly rapid mixed in a beaker and then poured into a $500 \mathrm{ml}$ graduated cylinder. After quickly and repeatedly inverted for 4 times, the height of the solid-liquid surface with time was immediately recorded until the height remained unchanged. Finally, the sludge flocs were collected for size measurement using a laser diffraction instrument (Mastersizer 2000, Malvern, U.K.). The measurement of each sample was repeated conducted for three times, and the average value was taken as the final value for analysis.

\section{Results and discussion}

\section{Structure identification}

FTIR spectra of polymers. Fig. 1 shows the FTIR spectra of AM, template PAMA (template poly(AM-MAPTAC)) and PMA (poly(MAPTAC)). As shown in Fig. 1 (AM), the absorption peaks at $3354,1675,1613,3189$ and $2813 \mathrm{~cm}^{-1}$ were assigned to stretching vibration of $\mathrm{N}-\mathrm{H}, \mathrm{C}=\mathrm{O}$ and $\mathrm{C}=\mathrm{C}$; asymmetry

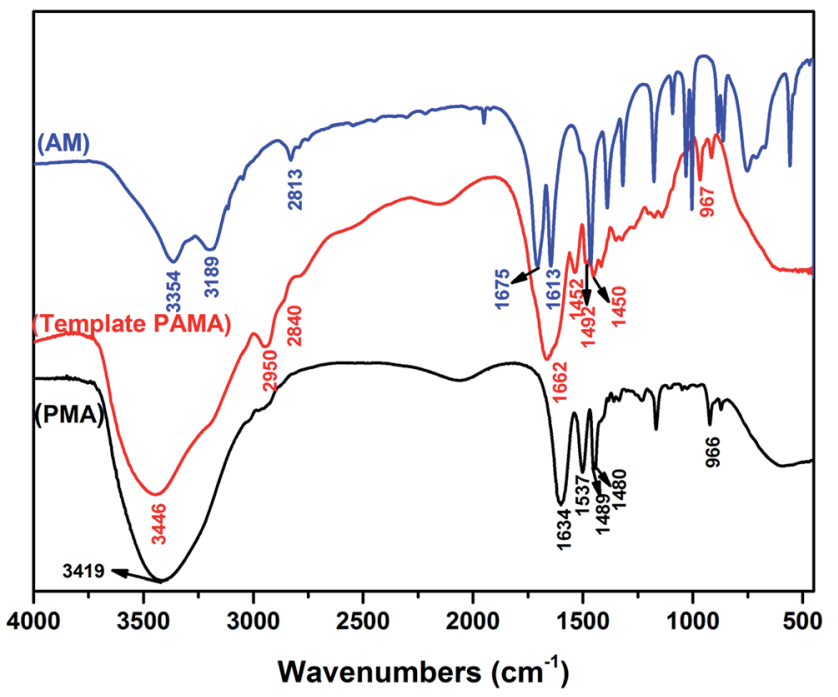

Fig. 1 FTIR spectra of polymers.

stretching vibration of $\mathrm{H}-\mathrm{C}=$ and symmetric stretching vibration of $\mathrm{H}-\mathrm{C}=.^{22,23}$ As shown in Fig. 1 (PMA), the absorption peaks at 3419, 2942, 1634, 1537, 1489, 1480 and $966 \mathrm{~cm}^{-1}$ were assigned to stretching vibration of $\mathrm{N}-\mathrm{H},-\mathrm{CH}_{3}, \mathrm{C}=\mathrm{O}, \mathrm{N}-\mathrm{H}$ and methyl groups of quaternary ammonium; deformation vibration of the methylene group in MAPTAC unit and $\mathrm{N}^{+}\left(\mathrm{CH}_{3}\right)_{3}$ stretching vibration. ${ }^{24}$ Comparing with Fig. 1 (AM) and (PMA), as shown in Fig. 1 (template PAMA), the peaks at 3189, 2813 and $1613 \mathrm{~cm}^{-1}$ which represent $\mathrm{C}=\mathrm{C}$ in $\mathrm{AM}$ disappeared; the peaks at 2942, 1489, 1480 and $966 \mathrm{~cm}^{-1}$ which represent MAPTAC unit were observed and the peaks at 3354(3419) and 1675(1634) which represent $\mathrm{N}-\mathrm{H}$ and $\mathrm{C}=\mathrm{O}$ both in $\mathrm{AM}$ and MAPTAC unit were also observed. All the corresponding peaks in template PAMA had a certain shift comparing with those in AM and MAPTAC. Therefore, all these characteristic peaks confirmed that the two monomers were successfully copolymerized.

${ }^{1} \mathbf{H}$ NMR of polymers. The ${ }^{1} \mathrm{H}$ NMR spectra of PMA, template PAMA and PAM are shown in Fig. 2. Comparing with the ${ }^{1} \mathrm{H}$

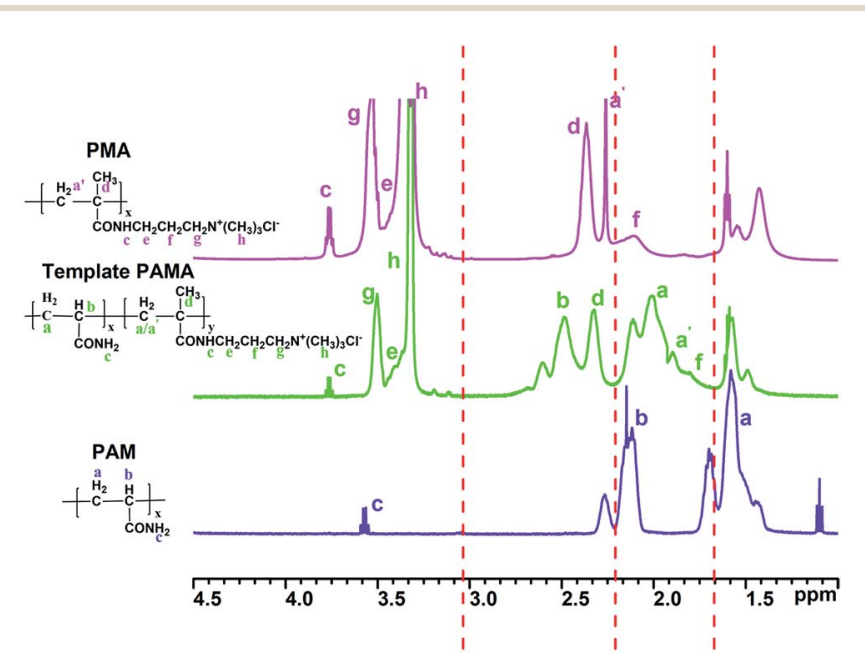

Fig. $2{ }^{1} \mathrm{H}$ NMR spectra of polymers. 


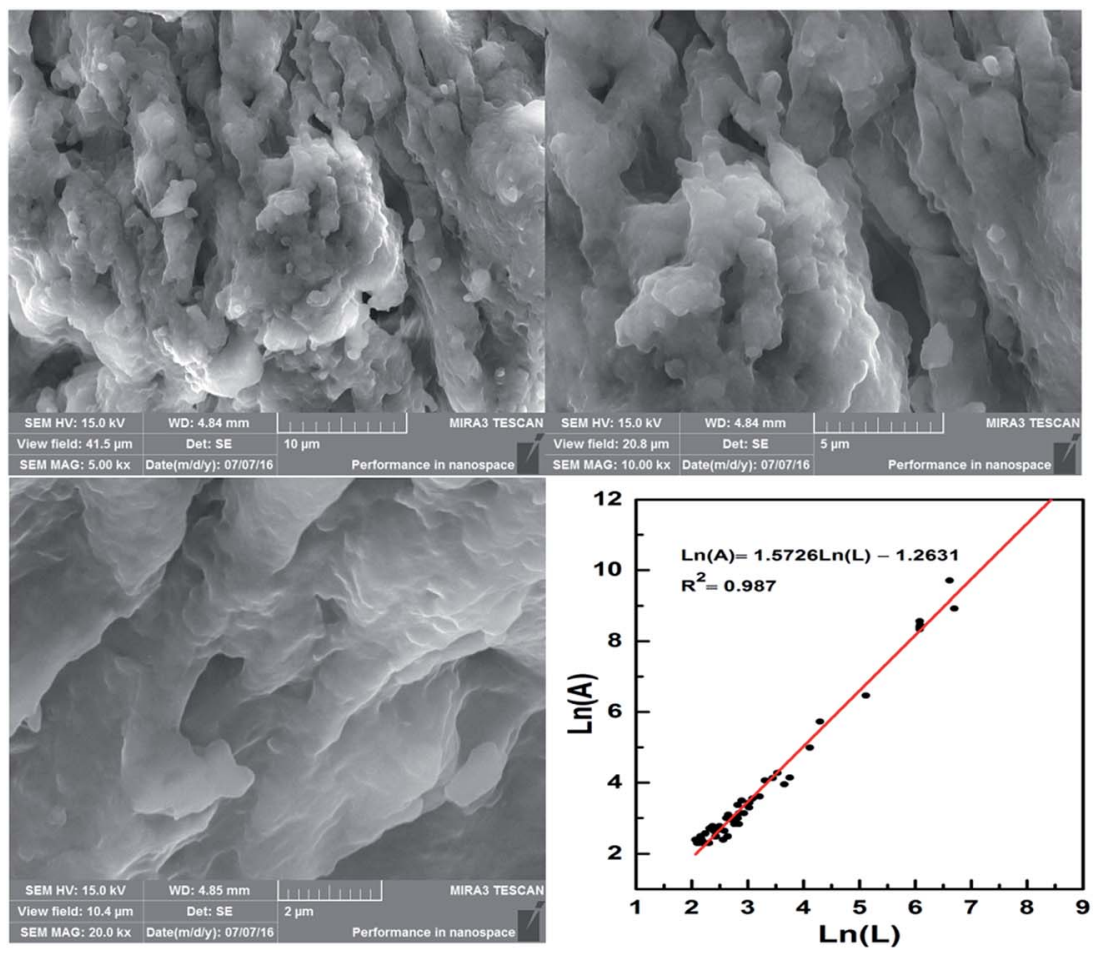

Fig. 3 SEM micrographs of template PAMA.

NMR spectra of PMA and PAM, the corresponding peaks in ${ }^{1} \mathrm{H}$ NMR spectra of template PAMA had a certain shift. The characteristic peaks of $\mathrm{H}_{\left(\mathrm{a}^{\prime}\right)}$ and $\mathrm{H}_{(\mathrm{c})} \sim \mathrm{H}_{(\mathrm{h})}$ at 1.94, 3.66, 2.06, 3.27, 1.77, 3.39 and $3.17 \mathrm{ppm}$ in PMA respectively and the characteristic peaks of $\mathrm{H}_{(\mathrm{a})}$ and $\mathrm{H}_{(\mathrm{b})}$ at 1.16 and $1.79 \mathrm{ppm}$ in PAM were all observed in the spectra of template PAMA., ${ }^{8,24}$ The coexistence of characteristic peaks of $\mathrm{H}_{(\mathrm{a})}$ and $\mathrm{H}_{\left(\mathrm{a}^{\prime}\right)}$ resulted from the two link mode of 'MAPTAC-AM-' and '-MAPTAC-MAPTAC-' meanwhile demonstrated the existence of micro-block structure of MAPTAC. Therefore, the results of ${ }^{1} \mathrm{H}$ NMR analysis indicated the successfully copolymerization of AM and MAPTAC moreover the existence of micro-block structure of MAPTAC.

SEM of copolymers. The SEM images of template PAMA $(5.00 \mathrm{k} \times, 10.00 \mathrm{k} \times$ and $20.0 \mathrm{k} \times)$ are shown in Fig. 3. As shown in Fig. 3, template PAMA had a porous structure with a prodigious surface area which could improve its ability of adsorption and bridging. This surface features should owe to the UV initiation mode with surface modification and removal of template PAAS. ${ }^{25,26}$ Ultraviolet radiation can induce the activation of carbon fiber surface and generation of free radicals. The free radicals react with monomer radicals to form chemical bond thus increased the surface energy and surface roughness (which usually shown as the increase of surface porosity) of carbon fiber. Some existing reports showed that the surface roughness of carbon fiber modified by UV induction mode had been increased which usually shown as the increase of surface porosity. Furthermore, in template PAMA without template separation, the template occupies a certain spatial location. Then with the removal of template, a certain pore formed at the original position of the template, thus porosity of template
PAMA was greatly increased. Besides the direct observation of SEM images, the fractal dimension $(D)$ of polymer calculated through image analysis method on the basis of SEM images can also be used to evaluate the surface morphology of polymer. ${ }^{27,28}$ Fractal dimension which corresponds to the complexity of the system is an important parameter in the study of fractal theory. In this study, Image-Pro Plus 6.0 software had been used to analysis the SEM images of template PAMA and fractal dimension used in this study was two-dimensional fractal dimension defined as double logarithmic relationship of perimeter $(L)$ and projected area $(A)$ of selected region. As shown in Fig. 3, log linear fitting of $L$ and $A$, the average fractal dimensions of template PAMA was 1.5726 which was much higher than that of

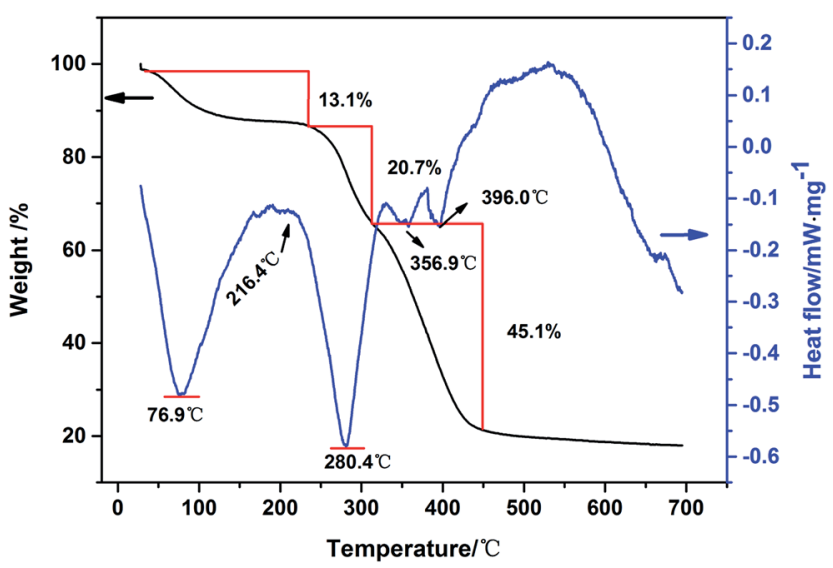

Fig. 4 TG/DSC analysis of template PAMA. 
Table 3 Variance analysis of regression model

\begin{tabular}{|c|c|c|c|c|c|c|}
\hline Source & Sum of squares & $\mathrm{df}$ & Mean square & $F$ value & $P$ value Prob $>F$ & \\
\hline Model & 102.78 & 20 & 5.14 & 34.35 & $<0.0001$ & Significant \\
\hline$X_{1}$ & 6.38 & 1 & 6.38 & 42.65 & $<0.0001$ & \\
\hline$X_{3}$ & 0.57 & 1 & 0.57 & 3.83 & 0.0616 & \\
\hline$X_{4}$ & 5.92 & 1 & 5.92 & 39.58 & $<0.0001$ & \\
\hline$X_{5}$ & 10.48 & 1 & 10.48 & 70.02 & $<0.0001$ & \\
\hline$X_{1} X_{4}$ & 0.37 & 1 & 0.37 & 2.5 & 0.1266 & \\
\hline$X_{1} X_{5}$ & 1.76 & 1 & 1.76 & 11.73 & 0.0021 & \\
\hline$X_{2} X_{3}$ & 0.037 & 1 & 0.037 & 0.25 & 0.624 & \\
\hline$X_{2} X_{4}$ & 0.51 & 1 & 0.51 & 3.43 & 0.076 & \\
\hline$X_{2} X_{5}$ & 0.11 & 1 & 0.11 & 0.71 & 0.4059 & \\
\hline$X_{3} X_{4}$ & 0.38 & 1 & 0.38 & 2.57 & 0.1215 & \\
\hline$X_{3}^{2}$ & 2.45 & 1 & 2.45 & 16.36 & 0.0004 & \\
\hline$X_{4}^{2}$ & 8.76 & 1 & 8.76 & 58.52 & $<0.0001$ & \\
\hline$X_{5}^{2}$ & 47.05 & 1 & 47.05 & 314.46 & $<0.0001$ & \\
\hline Residual & 3.74 & 25 & 0.15 & & & \\
\hline Lack of fit & 3.27 & 20 & 0.16 & 1.74 & 0.2806 & Not significant \\
\hline Pure error & 0.47 & 5 & 0.094 & & & \\
\hline Cor total & 106.52 & 45 & & & & \\
\hline
\end{tabular}

polymer flocculants synthesized by conventional method $\left(1.220^{8}, 1.300^{8}, 0.724^{3}, 0.714^{3}\right)$ also indicated a highly jumbled surface morphology.

TG/DSC analysis of copolymers. Fig. 4 shows the TG/DSC analysis of template PAMA. As shown in TG curve of Fig. 4. There were three stages of weight loss. Because of the hydrophilic groups in polymer chain, the powder sample was easy to absorb moisture in the air, and as the temperature increased, this part of the moisture began to evaporate. As a result, the strong endothermic peak at the first weight loss stage was observed at about $76.9^{\circ} \mathrm{C}$ in the DSC curve, and $13.1 \%$ of weight loss was calculated in this stage. The second stage of weight loss was considered for the thermal decomposition of main chemical functional groups such as amide and quaternary ammonium in polymer chain. ${ }^{29}$ The corresponding endothermic peak at $280.4{ }^{\circ} \mathrm{C}$ was observed and $20.7 \%$ of weight loss was calculated. Polymer backbone began to thermal decompose at the third stage $\left(311.3-456.3{ }^{\circ} \mathrm{C}\right)$ with a rapid weight loss of $45.1 \%$. Obviously, the two observed endothermic peak at $356.9^{\circ} \mathrm{C}$ and $396.0^{\circ} \mathrm{C}$ respectively indicated the coexist of two phase structure in the polymer and the existence of MAPTAC micro-block structure was verified from another point of view. ${ }^{20,30}$

\section{Optimize synthesis conditions by RSM}

The regression model of intrinsic viscosity affected by $\mathrm{pH}$, concentration of initiator, mole ratio of PAAS and MAPTAC, mole ratio of MAPTAC and AM and irradiation time were calculated using the least square method with the experimental data ( $\mathrm{S} 1$ Table $\dagger$ ). The final regression equation in terms of coded factors and the detail of variance analysis of the regression model are shown as eqn (2) and listed in Table 3 respectively. As shown in Table 3 , the $F$-value is 34.35 , and $p$ value (Prob $>F$ ) is less than 0.05 indicating statistically significance of this model. At the same time, $R^{2}$-value of 0.9648 suggested a relatively strong correlation between the observed and predicted values. This model could be used to analyze and predict the optimal conditions for the synthesis of template PAMA by UV-initiated template copolymerization. Furthermore, $X_{1}, X_{2}, X_{4}, X_{5}, X_{1} X_{2}, X_{1} X_{5}, X_{1}^{2}, X_{2}{ }^{2}, X_{3}^{2}, X_{4}{ }^{2}, X_{5}{ }^{2}$ were significant model terms, and the order of significant effect were irradiation time $(t / \mathrm{min})\left(X_{5}\right)>\mathrm{pH}\left(X_{1}\right)>$ mole ratio of MAPTAC and AM $\left(X_{4}\right)$ $>$ concentration of initiator $\left(X_{2}\right)$.

$$
\begin{aligned}
{[\eta]=} & 13.25+0.63 X_{1}+0.57 X_{2}-0.19 X_{3}+0.61 X_{4}+0.81 X_{5} \\
& +0.71 X_{1} X_{2}+0.33 X_{1} X_{3}+0.31 X_{1} X_{4}+0.66 X_{1} X_{5} \\
& +0.096 X_{2} X_{3}+0.36 X_{2} X_{4}+0.16 X_{2} X_{5}+0.31 X_{3} X_{4} \\
& +0.32 X_{3} X_{5}-0.058 X_{4} X_{5}-1.52 X_{1}^{2}-1.90 X_{2}^{2} \\
& -0.53 X_{3}^{2}-1.00 X_{4}^{2}-2.32 X_{5}^{2}
\end{aligned}
$$

Response surface analysis. Fig. 5 illustrates the interaction effect of $X_{1}(\mathrm{pH})$ and $X_{2}$ (concentration of VA-044) ((a) and (c)); $X_{1}(\mathrm{pH})$ and $X_{5}$ (irradiation time) ((b) and (d)) on the intrinsic viscosity of polymer. As shown in Fig. 5a, intrinsic viscosity of polymer showed a trend of first increasing and then decreasing along with the increase of concentration of VA-044, and the effect of $\mathrm{pH}$ on intrinsic viscosity also showed the same trend. As shown in the contour plot of Fig. 5c, pH showed more significant effect on intrinsic viscosity of polymer than concentration of VA-044, moreover, the interaction between $\mathrm{pH}$ and concentration of VA-044 affected the response 


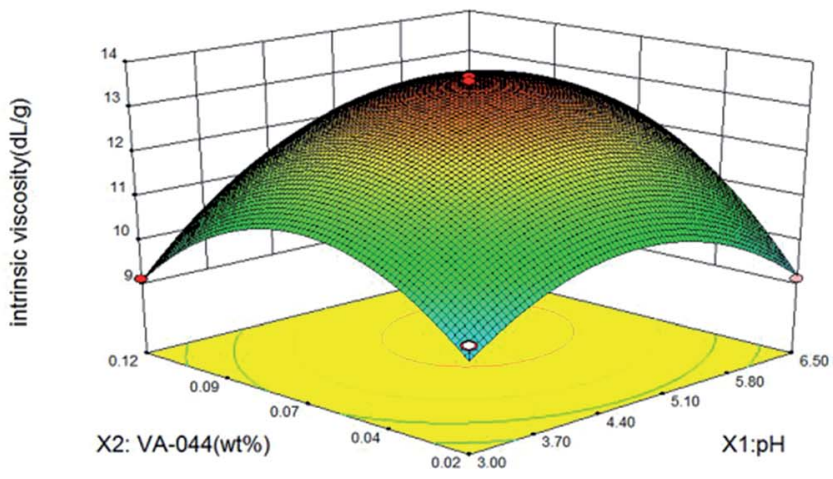

(a)

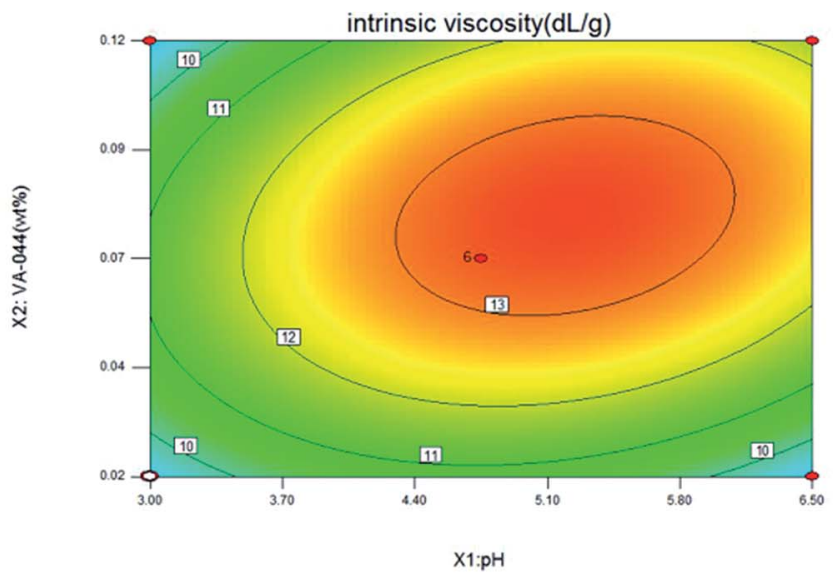

(c)

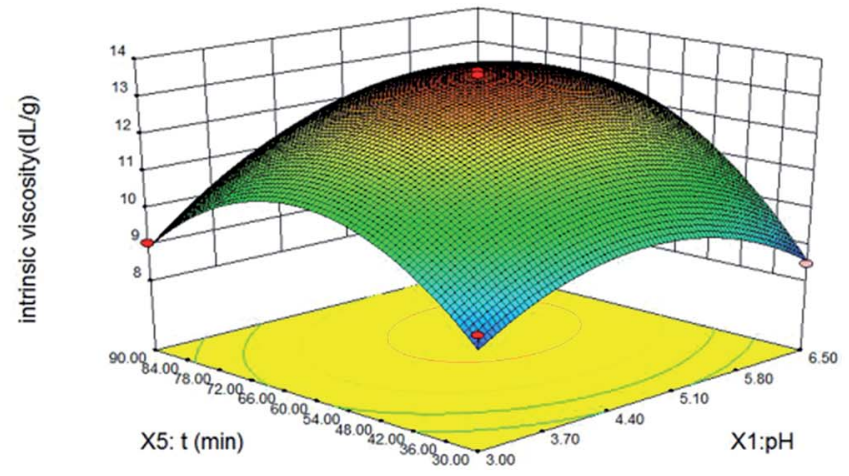

(b)

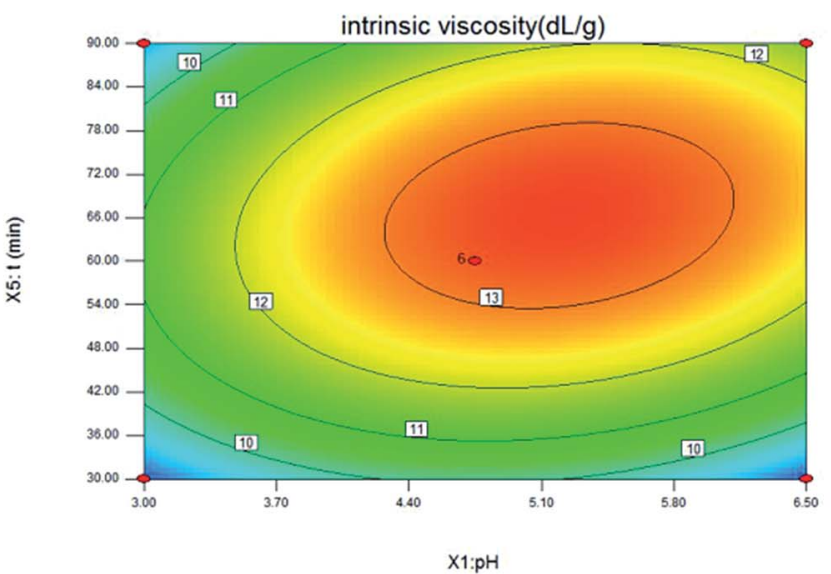

(d)

Fig. 5 RSM analysis of interaction effect ((a) and (c) interaction effect of $X_{1}(\mathrm{pH})$ and $X_{2}$ (concentration of VA-044); (b) and (d) interaction effect of $X_{1}(\mathrm{pH})$ and $X_{5}$ (irradiation time)).

obviously. Similarly, the effect of irradiation time showed the same trend on intrinsic viscosity of polymer with the concentration of VA-044 (Fig. 5b). However, the irradiation time showed more significant effect on intrinsic viscosity of polymer than $\mathrm{pH}$ and the interaction between $\mathrm{pH}$ and irradiation time also affected the response obviously. The other 8 interaction effect of factors were no significant indicated from Table 3.

Optimization and validation. RSM was used to search for an optimum combination of $\mathrm{pH}$, concentration of initiator, mole ratio of PAAS and MAPTAC, mole ratio of MAPTAC and AM and irradiation time for obtaining polymer with the maximum intrinsic viscosity. The results showed that the predicted intrinsic viscosity of $13.246 \mathrm{dL} \mathrm{g}^{-1}$ would be achieved when the $\mathrm{pH}$, concentration of initiator, mole ratio of PAAS and MAPTAC, mole ratio of MAPTAC and AM and irradiation time were $4.75,0.07 \mathrm{wt} \%, 1.0,0.33$ and $60 \mathrm{~min}$, respectively. Five additional independent experiments were carried out under the optimum condition. The predicted value was $13.246 \mathrm{dL} \mathrm{g}^{-1}$ of intrinsic viscosity and the actual experimental mean value was $13.223 \mathrm{dL} \mathrm{g}^{-1}$, indicating that the optimum condition was appropriate.

\section{Dyeing sludge dewatering}

Evaluation of dewatering performance. Residual turbidity (RT) of supernatant and filter cake moisture content (FCMC) after flocculation process are the main and direct indicators to evaluate flocculation efficiency of flocculants. Generally, lower RT and FCMC reflect excellent flocculation efficiency. Furthermore, specific resistance to filtration (SRF) is widely used to evaluate the filtration performance of sludge, and lower SRF reflects better dewatering performance of sludge. The effect of flocculants dosage and $\mathrm{pH}$ of sludge are illustrated in Fig. 6. As shown in Fig. 6a and b, with the flocculants dosage increased, the RT, FCMC and SRF first decreased to reach the minimum and then increased. This trend was consistent with the typical behavior of polyelectrolyte in the flocculation process reported in the previous literature. ${ }^{31}$ Furthermore, in the dewatering process of dyeing sludge which featured complex composition, high content of harmful substances, COD of supernatant is also one of the important indicators of concern. Also shown in Fig. $6 \mathrm{~b}$, the COD removal rate first increased to the maximum and then slightly decreased along with the increasing of flocculants dosage, this trend was corresponding with the other 

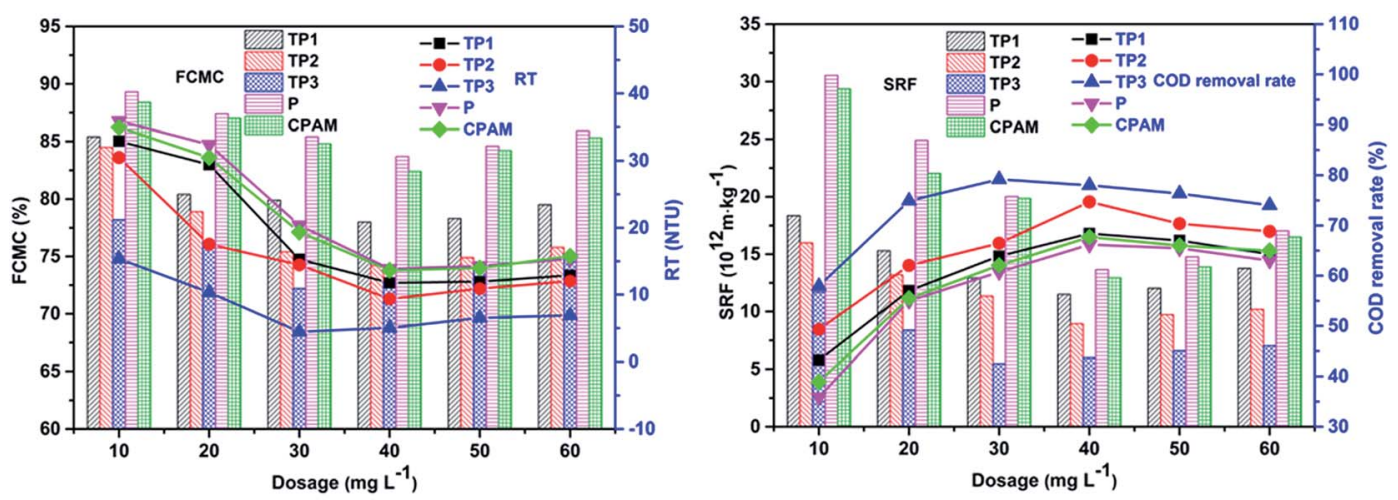

a
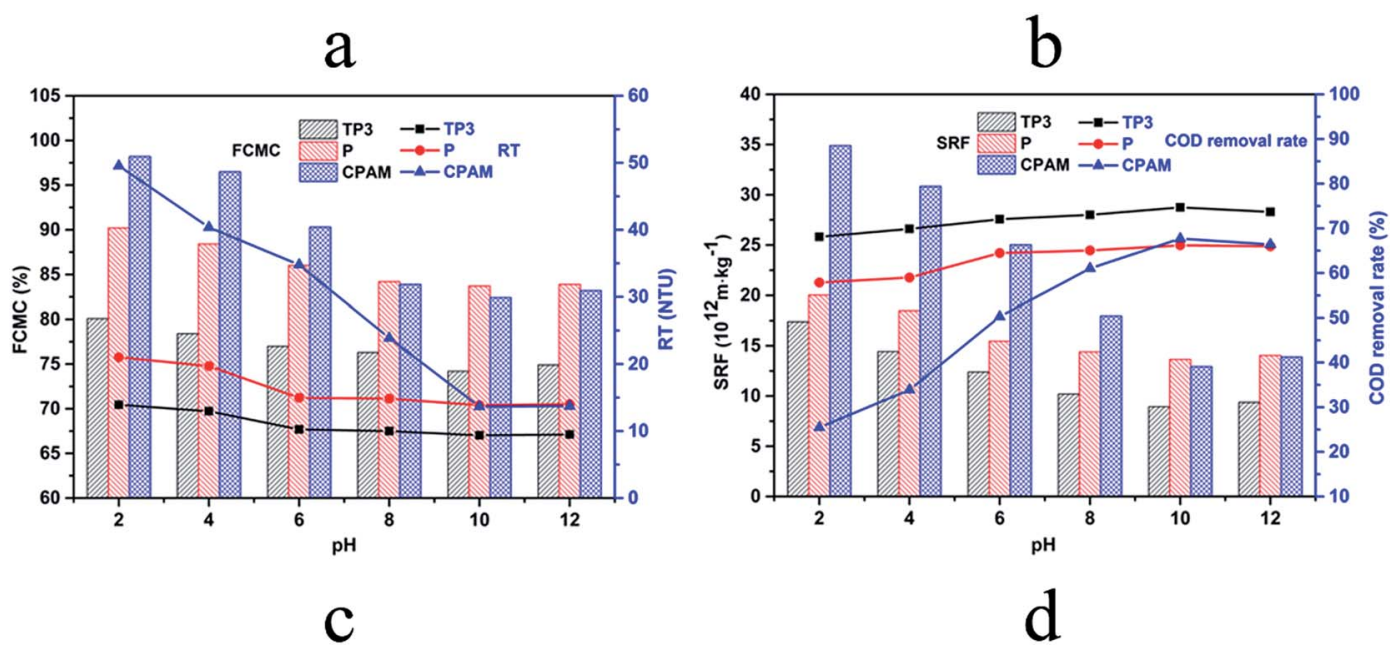

C

Fig. 6 Effect of flocculant dosage and pH of sludge on dewatering performance (experimental condition for a and b: $120 \mathrm{rpm}$ for $20 \mathrm{~s}, 40 \mathrm{rpm}$ for $60 \mathrm{~s}$, stand for $10 \mathrm{~min}$ and pH of 10.0; experimental condition for $\mathrm{c}$ and d: $120 \mathrm{rpm}$ for $20 \mathrm{~s}, 40 \mathrm{rpm}$ for $60 \mathrm{~s}$, stand for $10 \mathrm{~min}$ and dosage of TP3, P and CPAM were 30,40 and $40 \mathrm{mg} \mathrm{L}^{-1}$ ).

three indicators. The positively charged flocculants can neutralize the net charge on the surface of the negatively charged sludge, in addition, can reduce the surface tension of water and the thickness of the compressed double layer to reduce the thickness of the water film on the surface of the particle..$^{32}$ Due to the action of electric neutralization, the colloid particles in the sludge destabilized and gathered, correspondingly, the FCMC, RT and SRF were all decreased. Insufficient of electrical neutralization caused by low dosage of flocculants, electrostatic repulsion and steric effect caused by excess flocculants can cause sludge particles to re stable and not conducive to dewatering. ${ }^{33,34}$ Comparative analysis of the five flocculants, the TP3 presented the lowest RT, FCMC, SRF (4.47 NTU, $72.2 \%, 5.47 \times 10^{12} \mathrm{~m} \mathrm{~kg}^{-1}$ ) and highest COD removal rate (79.2\%) at a lower dosage of $30 \mathrm{mg} \mathrm{L}^{-1}$.

Compare analysis of TP1, TP2 and TP3 which featured the same cationic degree but different intrinsic viscosity, the dewatering efficiency of flocculants derived from the analysis of the indicators were enhanced with the increase of viscosity. This phenomenon indicated that in the sludge conditioning process by this kind of flocculants, bridging effect also played a crucial role. In the process of bridging, the polymer chains can span and reduce the gap between the colloidal particles to make the colloid destabilized and gathered to settle. Polymer with higher intrinsic viscosity feature longer molecular chain, consequently improve the collision probability between flocculants and sludge particle, which is conducive to the trapping and bridging action for colloidal particle. ${ }^{35}$ Therefore, to improve the intrinsic viscosity of flocculants is one of the important means to enhance the flocculation efficiency. In addition, these results indicated the significance of RSM optimize section in this paper.

In view of the importance of sludge $\mathrm{pH}$ in the flocculation process and the $\mathrm{pH}$ diversity of sludge to be treated, the study of relationship between the dewatering performance and the $\mathrm{pH}$ value of sludge is of great practical significance. The effect of sludge $\mathrm{pH}$ on dewatering performance is illustrated in Fig. 6c and $\mathrm{d}$. With the increased $\mathrm{pH}$, after sludge treated by CPAM, the RT, FCMC and SRF sharply decreased to the minimum (13.66 NTU, $82.4 \%$ and $12.93 \times 10^{12} \mathrm{~m} \mathrm{~kg}^{-1}$ ) at $\mathrm{pH} 10.0$, in addition, the COD removal rate sharply increased to the maximum (67.7\%) accordingly. The results indicated that alkaline conditions were more favorable for CPAM to exert flocculation efficiency in the process of dyeing sludge dewatering. However, as shown in Fig. 6c and d, comparing with CPAM, after treated by TP3 and P, the change of the four indicators was slight. This phenomenon could be explained by the high stability of polymer chain of TP3 and P under acid/base condition which could 

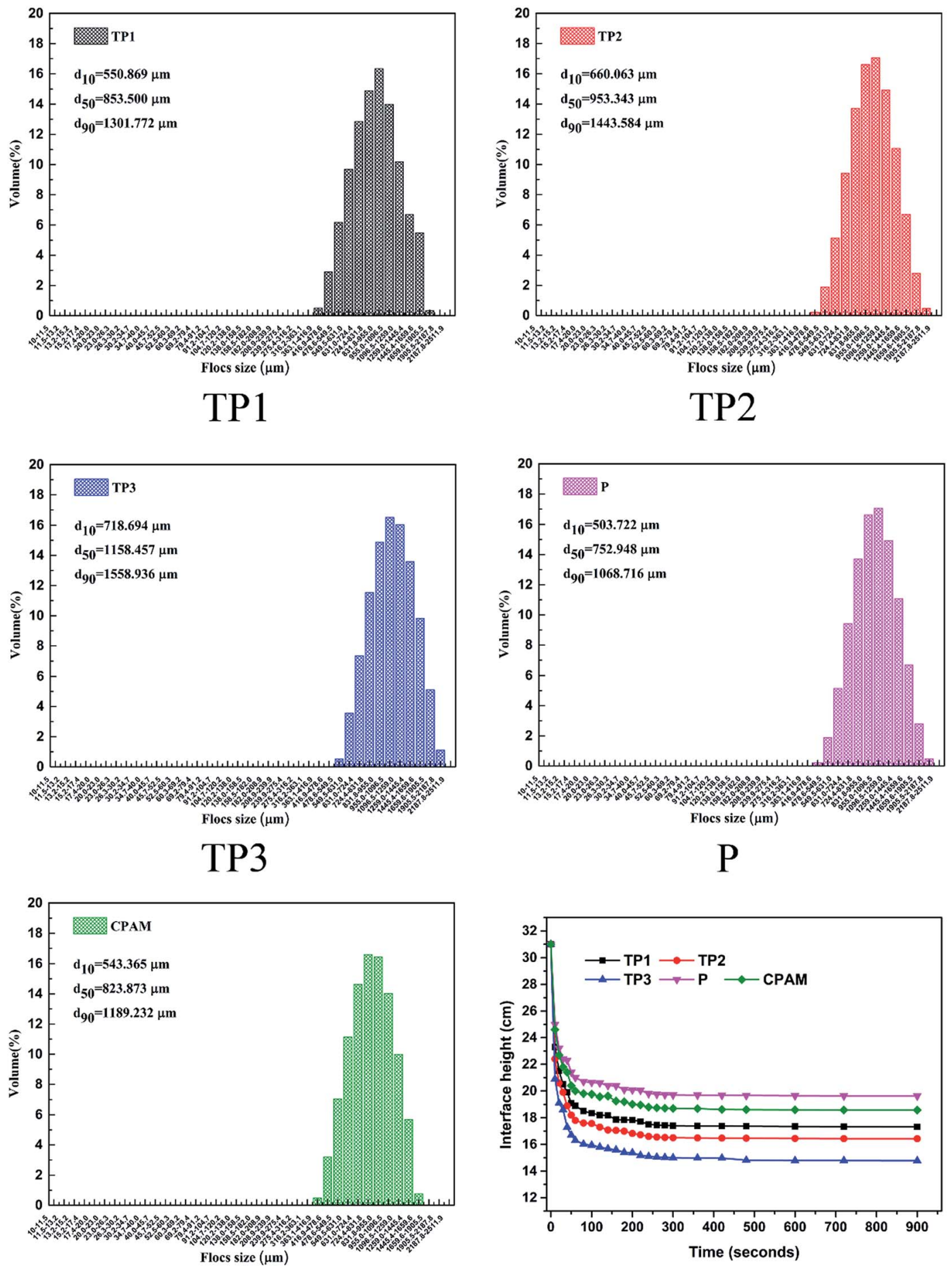

CPAM

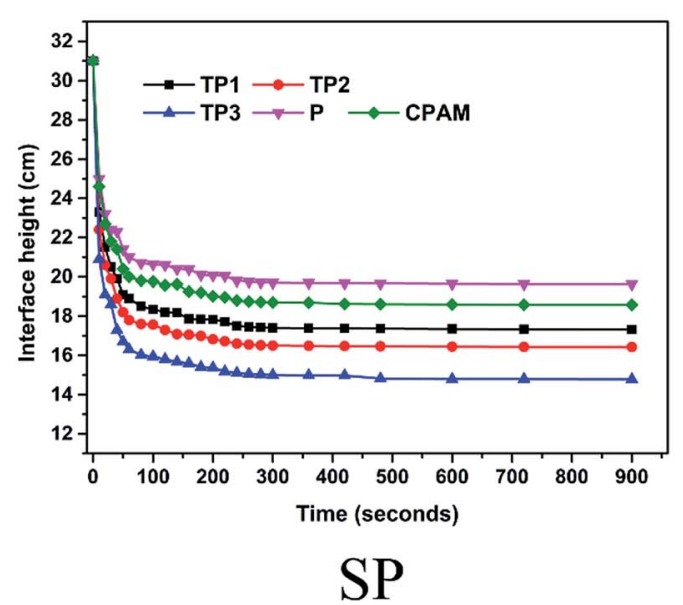

Fig. 7 Sludge floc size distribution at the end of flocculation process and effect of types of flocculants on sludge settling behaviors (experimental condition: dosage of TP1, TP2, TP3, P and CPAM were 40, 40, 30, 40 and $40 \mathrm{mg} \mathrm{L}^{-1} ; \mathrm{pH}$ of 10.0).

attribute the success to the stable amide bond in the TP3 and P molecular chain instead of acyl oxygen bond in the CPAM molecular chain.

Sludge flocs properties and flocculation mechanism. Sludge settle performance is often regarded as one of the indexes to evaluate the sludge dewatering. Sludge with better settle performance usually has better dewatering performance. ${ }^{36}$ Generally, flocs size and density in particular, can influence sludge settling performance significantly. ${ }^{37}$ Fig. 7 SP illustrates the sludge settling behaviors after treated by the five flocculants. Results showed that all the sludge settling had a first fast (within 100 seconds), then slow (within 500 seconds) and steady 


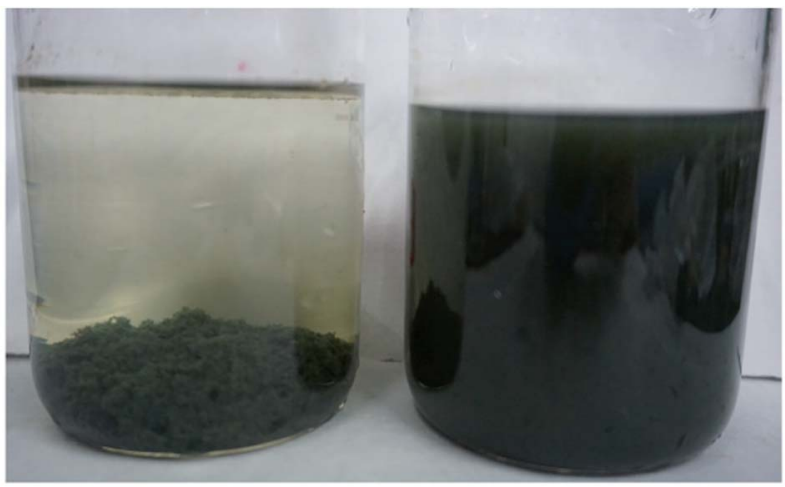

After

Before

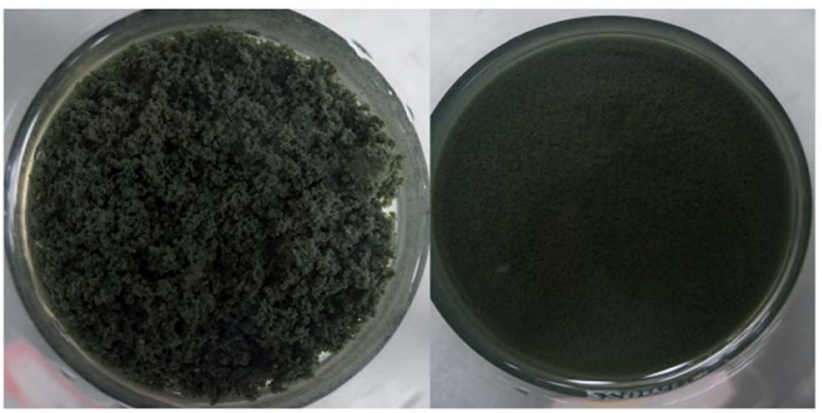

After

Before

Fig. 8 Comparison chart before and after flocculation for TP3 $(30 \mathrm{mg}$ $\mathrm{L}^{-1}$ of dosage, 10.0 of $\mathrm{pH}$ ).

(within 900 seconds) process. The average sedimentation rates of the fast process were $0.1266,0.1343,0.1506,0.1036$ and $0.1124 \mathrm{~cm} \mathrm{~s}^{-1}$ for TP1, TP2, TP3, P and CPAM respectively. In addition, the final sediment height which indicated the sludge volume after dewatering process were 17.321, 16.430, 14.924, 19.605 and $18.714 \mathrm{~cm}$ for TP1, TP2, TP3, P and CPAM respectively. These results indicated that TP3 had the best settle performance among the test flocculants and with the increase of intrinsic viscosity, the settle performance enhanced. In addition, we investigated the flocs size distribution (showed in Fig. 7) which represented another important influence factor of sludge settle performance for the test flocculants. As shown in Fig. 7, the $d_{50}$ of flocs after treated by the five flocculants was in the following order: TP3 $>$ TP2 $>$ TP1 $>$ CPAM $>$ P. These results also indicated the better settle performance of TP3. Fig. 8 shows the treat effect of TP3 on sludge intuitively. After flocculation process, fine sludge particles formed large and dense flocs and settled to the bottom of the beaker with clarified supernatant. As a result, all the conclusion obtained from flocs properties was in line with the former dewatering performance evaluation indicators.

Adsorption bridging and charge neutralization are the main mechanism of flocculation process. In addition, zeta potential of flocculation system can verify the charge neutralization effect directly and effectively. ${ }^{38}$ Fig. 9 illustrates the zeta potential of flocculation system along with the increasing of flocculants dosage. All the zeta potential of system after flocculation process were increased with the increased dosage. This

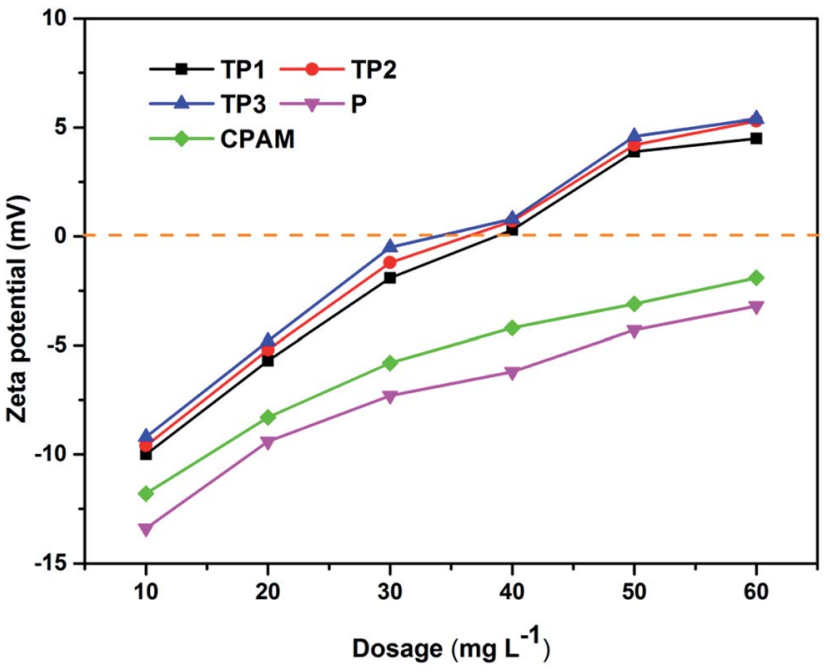

Fig. 9 Effect of dosage on zeta potential (experimental condition: dosage of TP1, TP2, TP3, P and CPAM were 40, 40, 30, 40 and $40 \mathrm{mg}$ $\mathrm{L}^{-1} ; \mathrm{pH}$ of 10.0).

phenomenon indicated charge neutralization played an important role in the flocculation process. Zeta potential of TP1, TP2 and TP3 at their optimum dosage were all close to zero indicated charge neutralization was the mainly flocculation mechanism..$^{39}$ However, zeta potential of P and CPAM at their optimum dosage were all below zero indicated that charge neutralization played a less important role than that of TP1, TP2 and TP3. Furthermore, TP1, TP2 and TP3 had the almost same of zeta potential which can be considered as the same charge neutralization effect, yet with different dewatering performance. This phenomenon could attribute to the different intrinsic viscosity of polymer which could affect the adsorption bridging effect in flocculation process.

\section{Conclusions}

In this research, UV-initiated template copolymerization technology was used to prepare cationic polyacrylamide with microblock structure. All the results of structural characterization verified the successful synthesis and existence of micro-block structure. Furthermore, in order to promote its molecular weight, Box-Behnken experiment design and Respond Surface Method (RSM) were employed to optimum the synthesis conditions taking intrinsic viscosity as the response factor. The optimum synthesis conditions were 4.75 for $\mathrm{pH}, 0.07 \mathrm{wt} \%$ for initiator concentration, 1.0 for mole ratio of PAAS and MAPTAC, 0.33 for mole ratio of MAPTAC and AM, and $60 \mathrm{~min}$ for irradiation time respectively, and under these conditions, about $13.223 \mathrm{dL} \mathrm{g}^{-1}$ of intrinsic viscosity can be obtained. Results of dyeing sludge dewatering tests showed that the TP3 with microblock structure exhibited excellent dewatering performance and at $30 \mathrm{mg} \mathrm{L}^{-1}$ of dosage, and $10.0 \mathrm{of} \mathrm{pH}$, the RT, FCMC, SRF and COD removal rate reached the optimum of $4.47 \mathrm{NTU}, 72.2 \%$, $5.47\left(10^{12} \mathrm{~m} \mathrm{~kg}^{-1}\right)$ and $79.2 \%$ respectively; in the range below the optimum intrinsic viscosity obtained in this research, the 
dewatering performance was promoted along with the increased intrinsic viscosity; TP series of flocculants which synthesized taking MAPTAC as the cation monomer had a wider range of $\mathrm{pH}$ application than DAC series of flocculants (CPAM).

\section{Abbreviations}

$\begin{array}{ll}\text { AM } & \text { Acrylamide } \\ \text { MAPTAC } & \begin{array}{l}\text { Methacrylamido propyl trimethyl ammonium } \\ \text { chloride }\end{array} \\ \text { DAC } & \text { Acryloyloxyethyltrimethylammonium chloride } \\ \text { CPAM } & \text { Cationic polyacrylamide } \\ \text { UV } & \text { Ultraviolet } \\ \text { PAAS } & \text { Sodium polyacrylate } \\ \text { VA-044 } & \text { 2,2'-Azobis[2-(2-imidazolin-2-yl)propane] } \\ & \text { dihydrochloride } \\ { }^{1} \mathrm{H} \text { NMR } & { }^{1} \text { H nuclear magnetic resonance } \\ \text { FTIR } & \text { Fourier transform-infrared spectroscopy } \\ \text { SEM } & \text { Scanning electron microscopy } \\ \text { TG/DSC } & \text { Thermogravimetric/differential scanning calorimetry } \\ \text { RT } & \text { Residual turbidity } \\ \text { FCMC } & \text { Filter cake moisture content } \\ \text { SRF } & \text { Specific resistance to filtration } \\ \text { TP(1-3) } & \text { Template poly(AM-MAPTAC)(1-3) } \\ \text { P } & \text { Poly(AM-MAPTAC) } \\ \text { COD } & \text { Chemical oxygen demand } \\ \text { RSM } & \text { Response surface analysis } \\ M_{\mathrm{W}} & \text { Molecular weight } \\ \text { CD } & \text { Cationic degree } \\ & \end{array}$

\section{Acknowledgements}

This research was supported by the National Natural Science Foundation of China (Project No. 21477010) and 111 Project (Project No. B13041).

\section{References}

1 H. J. Luo, X. A. Ning, X. J. Liang, Y. F. Feng and J. Y. Liu, Bioresour. Technol., 2013, 139, 330-336.

2 X. A. Ning, Y. F. Feng, J. J. Wu, C. M. Chen, Y. J. Wang, J. Sun, et al., J. Environ. Manage., 2015, 162, 81-86.

3 H. L. Zheng, Y. J. Sun, C. J. Zhu, J. S. Guo, C. Zhao, Y. Liao, et al., Chem. Eng. J., 2013, 234, 318-326.

4 J. R. Zhu, H. L. Zheng, Z. Z. Jiang, Z. Zhang, L. W. Liu and Y. J. Sun, Desalin. Water Treat., 2012, 51, 2791-2801.

5 Y. Qi, K. B. Thapa and A. F. A. Hoadley, Chem. Eng. J., 2011, 171, 373-384.

6 Y. Liao, H. L. Zheng, L. Qian, Y. J. Sun, L. Dai and W. W. Xue, Ind. Eng. Chem. Res., 2014, 53, 11193-11203.

7 Y. X. Zhao, B. Y. Gao, Q. B. Qi, Y. Wang, S. Phuntsho, J. H. Kim, et al., J. Hazard. Mater., 2013, 258-259C(6), 84-92. 8 H. L. Zheng, Y. J. Sun, J. S. Guo, F. Li, W. Fan, Y. Liao, et al., Ind. Eng. Chem. Res., 2014, 53, 2572-2582.

9 Y. J. Sun, W. Fan, H. L. Zheng, Y. X. Zhang, F. T. Li and W. Chen, PLoS One, 2015, 10(6), e0130683.
10 J. R. Zhu, H. L. Zheng, Z. Z. Jiang, Z. Zhang, L. W. Liu, Y. J. Sun, et al., Desalin. Water Treat., 2013, 51(13-15), 2791-2801.

11 X. G. Liu, Q. Chen, K. Xu, X. D. Zhang and P. X. J. Wang, Appl. Polym. Sci., 2009, 113(4), 2693-2701.

12 H. L. Zheng, Y. Liao, M. Z. Zheng, C. J. Zhu, F. Y. Ji, J. Y. Ma, et al., Sci. World J., 2014, 2014, 1-11.

13 B. Bolto and J. Gregory, Water Res., 2007, 41, 2301-2324.

14 Y. Zhou, G. J. Jameson and G. V. Franks, Colloids Surf., A, 2008, 331(3), 183-194.

15 Y. Zhou and G. V. Franks, Langmuir, 2006, 22(16), 6775-6786.

16 L. J. Wang, G. D. Li, Y. Q. Zhang and H. J. Xiao, Appl. Polym. Sci., 2013, 130(6), 4040-4046.

17 W. C. Chai, Y. W. Zhang and Y. F. Hou, Polym. Chem., 2013, 4(4), 1006-1013.

18 A. Q. Zhang, Y. H. Shen, X. Y. Zhang, L. C. Han and Z. Z. Wang, J. Polym. Res., 2009, 17(1), 11-17.

19 M. Ma and S. Zhu, Colloid Polym. Sci., 1999, 277(2-3), 123-129.

20 W. Chen, H. L. Zheng, Q. Q. Guan, H. K. Teng, C. Zhao and C. L. Zhao, Ind. Eng. Chem. Res., 2016, 55, 2892-2902.

21 Q. Q. Guang, H. L. Zheng, J. Zhai, B. Z. Liu, Y. J. Sun, Y. L. Wang, et al., J. Appl. Polym. Sci., 2015, 132, 41747.

22 P. Lanthong, R. Nuisin and S. Kiatkamjornwong, Carbohydr. Polym., 2006, 66, 229-245.

23 N. Şahiner, S. Malcı, Ö. Çelikbıçak, Ö. Kantoğlu and B. Salih, Radiat. Phys. Chem., 2005, 74, 76-85.

24 A. Pourjavadi, S. M. Fakoorpoor and S. H. Hosseini, Carbohydr. Polym., 2013, 93, 506-511.

25 K. H. Hong, N. Liu and G. Sun, Eur. Polym. J., 2009, 45, 24432449.

26 B. Kordoghli, R. Khiari, M. F. Mhenni, F. Sakli and M. N. Belgacem, Appl. Surf. Sci., 2012, 258, 9737-9741.

27 G. C. Zhu, H. L. Zheng, Z. Zhang, T. Tshukudu, P. Zhang and X. Y. Xiang, Chem. Eng. J., 2011, 178, 50-59.

28 H. L. Zheng, G. C. Zhu, S. J. Jiang, T. Tshukudu, X. Y. Xiang, P. Zhang, et al., Desalination, 2011, 269, 148-156.

29 L. Ghimici, I. Dranca, S. Dragan, T. Lupascu and A. Maftuleac, Eur. Polym. J., 2001, 37, 227-231.

30 Q. Q. Guan, H. L. Zheng, J. J. Zhai, C. Zhao, X. K. Zheng, X. M. Tang, et al., Ind. Eng. Chem. Res., 2014, 53, 5624-5635.

31 Z. Abdollahi, M. Frounchi and S. S. J. Dadbin, Ind. Eng. Chem., 2011, 17(3), 580-586.

32 J. P. Wang, S. J. Yuan, Y. Wang and H. Q. Yu, Water Res., 2013, 47(8), 2643-2648.

33 Z. Yang, H. Yang, Z. W. Jiang, T. Cai, H. J. Li, H. B. Li, et al., J. Hazard. Mater., 2013, 254, 36-45.

34 G. C. Zhu, H. L. Zheng, W. Y. Chen, W. Fan, P. Zhang and T. Tshukudu, Desalination, 2012, 285, 315-323.

35 M. Hjorth and B. U. Jørgensen, Water Res., 2012, 46(4), 10451051.

36 Y. G. Chen, Y. S. Chen and G. W. Gu, Chem. Eng. J., 2004, 99, 137-143.

37 A. R. Heath, P. A. Bahri, P. D. Fawell and J. B. Farrow, AIChE J., 2006, 52(6), 1987-1994.

38 B. Y. Gao, Y. Wang, Q. Y. Yue, J. C. Wei and Q. Li, Sep. Purif. Technol., 2007, 54, 157-163.

39 E. Pefferkorn, Adv. Colloid Interface Sci., 2006, 120, 33-45. 